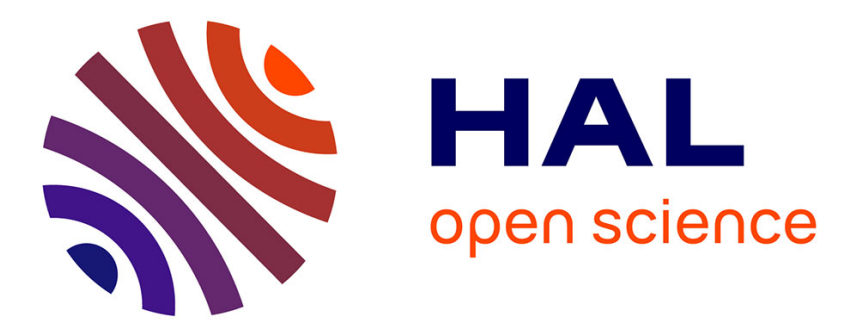

\title{
Hamiltonian point of view on parallel interconnection of buck converters
}

Jérémie Kreiss, Jean-François Trégouët, Damien Eberard, Romain Delpoux, Jean-Yves Gauthier, Xuefang Lin-Shi

\section{- To cite this version:}

Jérémie Kreiss, Jean-François Trégouët, Damien Eberard, Romain Delpoux, Jean-Yves Gauthier, et al. Hamiltonian point of view on parallel interconnection of buck converters. IEEE Transactions on Control Systems Technology, 2021, 29 (1), pp.43-52. 10.1109/TCST.2019.2961073 . hal-03377928

\section{HAL Id: hal-03377928 \\ https://hal.science/hal-03377928}

Submitted on 5 Nov 2021

HAL is a multi-disciplinary open access archive for the deposit and dissemination of scientific research documents, whether they are published or not. The documents may come from teaching and research institutions in France or abroad, or from public or private research centers.
L'archive ouverte pluridisciplinaire HAL, est destinée au dépôt et à la diffusion de documents scientifiques de niveau recherche, publiés ou non, émanant des établissements d'enseignement et de recherche français ou étrangers, des laboratoires publics ou privés. 


\title{
Hamiltonian Point of View on Parallel Interconnection of Buck Converters
}

\author{
Jérémie Kreiss ${ }^{1}$, Jean-François Trégouët ${ }^{1}$, Damien Eberard ${ }^{1}$, \\ Romain Delpoux ${ }^{1}$, Jean-Yves Gauthier ${ }^{1}$ and Xuefang Lin-Shi ${ }^{1}$
}

\begin{abstract}
In this paper, parallel interconnection of DC/DC converters is considered. For this topology of converters feeding a common load, it has been recently shown that dynamics related to voltage regulation can be completely separated from the current distribution without considering frequency separation arguments, which inevitably limits achievable performance. Within the Hamiltonian framework, this paper shows that this separation between current distribution and voltage regulation is linked to the energy conservative quantities: the Casimir functions. Furthermore, a robust control law is given in this framework to get around the fact that the load might be unknown. In this paper, we also ensure that the system converges to the optimal current repartition, without requiring explicit expression of the optimal locus. Finally, resulting control law efficiency is assessed through experimental results.
\end{abstract}

\section{INTRODUCTION}

Nowadays, many applications such as lowvoltage/high-current power supplies are composed of several power converters connected to a single load. Indeed, this structure benefits from several advantages as a consequence of the free distribution of load current on each converter. Thereby, it is possible to increase reliability, ease of repair, improve thermal management or reduce output ripple by interleaving phase of Pulse Width Modulation (PWM) for example.

The main challenge on this kind of structure is to regulate output voltage and current distribution together which are coupled dynamics. To cope with this difficulty, most of existing solutions (see e.g. [1], [2], [3]) propose control design procedure based on frequency consideration that separates dynamics of the system (output voltage from current distribution). Inevitably, those considerations reduce the achievable performance by imposing slow current distribution dynamics.

\footnotetext{
${ }^{1}$ Every author is with Laboratoire Ampère, INSA Lyon, Université de Lyon, 20, Avenue Albert Einstein, 69100 Villeurbanne, France firstname. lastname@insa-lyon.fr. Corresponding author mail: jeremie.kreiss@insa-lyon.fr
}

However, new solutions have been recently presented [4] where the separation between voltage and current distribution dynamics is geometric. Indeed, by both state and input change of coordinates, those two dynamics are disconnected without any frequency considerations. Hence, this approach provides a framework to easily deal with the two dynamics without sacrificing performance for an arbitrary number of DC/DC buck converters with distinct characteristics.

In this paper, main result of [4] is considered in a different framework: the Port-ControlledHamiltonian (PCH) formalism (see [5] for more details, [6], [7] for power converters in this framework). In addition to describing a large class of non-linear models, the PCH structure intrinsically yields many interesting features such as: (i) energy conservative property, (ii) obvious decomposition between interconnection and damping elements, (iii) straightforward relation that link the dynamics to the energy of the system and (iv) attractive nature of interconnection on ports allowing some Plug\&Play behaviours. Furthermore the extension to more complicated converters, potentially non-linear, fits into the PCH frame.

Classical control design methods on PCH models, namely interconnection and damping assignment passivity-based control (IDA-PBC) introduced in [8] aim to stabilize the dynamics. Yet, the occurrence of disturbance, uncertainties or reference signal leads to steady-state errors and undesirable behaviour. As we consider that the load is unknown, which is the case in most of practical cases, we will suffer from this. That is why we resort to robust energy shaping control methods which are developed in the Hamiltonian formalism (see [9]). In practice, those methods reduce to the addition of an integral action on the PCH ports. Unfortunately, in our case, integral actions on the Hamiltonian ports are not sufficient to deal with uncertainties or disturbances. Yet, interesting developments have been provided in [10] and [11] where integral action on non-passive 
outputs is considered.

Main contributions of this paper are as follows: 1) Geometric decomposition proposed in [4] is completely revisited within the Hamiltonian framework. A new change of coordinates is proposed, which can be related to the presence of Casimir function. Here, as compared to [4], not only current repartition does not impact voltage regulation but the opposite also holds: Current distribution is made independent from voltage regulation. As a result, the system can be separated into two independent subsystems whereas only cascaded form was achieved in [4]. 2) Loadindependent controller is proposed and proved to comply with all control specifications, so that unknown load can be taken into account. Inspired by [10] and [11], where constant exogenous disturbance is considered, our result is derived relying on integral action on non-passive outputs via a constructive approach which applies to state dependent disturbance. 3) Optimal current repartition is achieved at the steady state. Specification about this secondary objective can be conveniently expressed as an optimization problem. Using strictly convex cost function satisfying some assumptions, we are able to design a controller which ensures that the equilibrium point is the minimum of the cost function even if this minimum is unknown. 4) As a last contribution, experiment results are provided.

The paper is structured as follows. In Section II, Hamiltonian model of the parallel converters is given as well as the control problem. Section III provides a useful change of coordinates related to Casimir functions in order to separate output voltage dynamics from current repartition; Original control problem is then rewritten in the new coordinates. Control design examples for both known and unknown load are described in Section IV. Finally, Section V presents some experimental results.

Notation: The notation $x_{k}$ refers to the $k$-th element of vector $x$, with 1 being the index of first element. Given a function $f: \mathbb{R}^{n} \rightarrow \mathbb{R}$ we define the operators

$\mathbb{R}^{n \times 1} \ni \nabla_{x} f:=\left(\frac{\partial f}{\partial x}\right)^{\top}, \quad \mathbb{R}^{p \times 1} \ni \nabla_{\xi} f:=\left(\frac{\partial f}{\partial \xi}\right)^{\top}$,

where $\xi \in \mathbb{R}^{p}$ is a sub-vector of the vector $x$. The symbol $\mathbf{I}_{m}$ stands for the identity matrix of size $m \times m$. The null matrix of size $m \times n$ is denoted by $\mathbf{0}_{m \times n}$. The vector (column matrix) of size $m$ for which every entry is 1 (respectively 0 ) is denoted by $\mathbf{1}_{m}$ (respectively $\mathbf{0}_{m}$ ). The operator "diag" builds

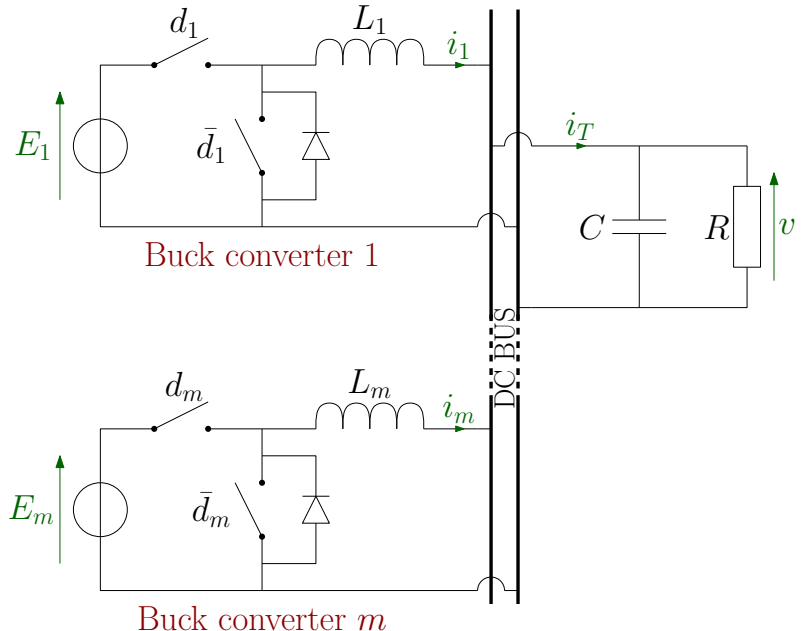

Fig. 1: Electrical schematic of $m$ Buck converters

diagonal matrix from entries of the input vector argument.

\section{Problem statement}

In this paper, we are interested in the electrical circuit shown in Fig. 1 which corresponds to parallel interconnection of $m$ heterogeneous and synchronous buck converters sharing a single capacitor $C$ and connected to a common resistive load $R$. Instead of acting directly on the switches and dealing with a hybrid system (like e.g. [7]), converters are controlled here via PWM where $d_{k}$ refers to duty cycle of the $k$-th converter and $\bar{d}_{k}=1-d_{k}$ is its complementary signal. Index $k$ belongs to the set $\{1, \ldots, m\}$. Capacitor charge is defined as $Q=C v$ where $v$ is DC bus voltage. Furthermore, we denote by $\varphi_{k}$ the magnetic flux in $k$-th inductor $L_{k}$ which is linked to the current $i_{k}$ by the relation $\varphi_{k}=L_{k} i_{k}$. $E_{k}$ corresponds to the voltage source of the $k$-th converter.Vector $L \in \mathbb{R}^{m}$ (resp. $\varphi, i, E \in \mathbb{R}^{m}$ ) gathers every element $L_{k}$ (resp. $\left.\varphi_{k}, i_{k}, E_{k}\right)$ for all $k \in\{1, \ldots, m\}$.

Throughout this paper, we assume that (i) switching frequency $f_{s}$ is sufficiently large for the dynamics to be approximated by an average continuous time model, and (ii) electrical components and switches are ideal, i.e. parasitic elements can be neglected.

Under those assumptions and using Kirchoff's laws on the energy variables, dynamics of circuit depicted in Fig. 1 are expressed by

$$
\begin{aligned}
\forall k \in\{1, \ldots, m\}, \frac{\mathrm{d} \varphi_{k}}{\mathrm{~d} t} & =-\frac{Q}{C}+E_{k} d_{k}, \\
\frac{\mathrm{d} Q}{\mathrm{~d} t} & =\sum_{k=1}^{m} \frac{\varphi_{k}}{L_{k}}-\frac{Q}{R C} .
\end{aligned}
$$


Eq. (1a) refers to the dynamics of inductors flux produced by each converters whereas (1b) describes the output capacitor charge dynamics.

This leads to the following linear Hamiltonian system (see [6])

$$
\dot{x}=(\mathcal{J}-\mathcal{R}) \nabla_{x} H(x)+B d
$$

where $\mathbb{R}^{m+1} \ni x=\left[\varphi^{\top}, Q\right]^{\top}$ gathers the energy variables and the smooth function

$$
H(x)=\frac{1}{2} x^{\top} \operatorname{diag}\{L, C\}^{-1} x
$$

represents the total stored energy. Energy dissipation is characterized by the $n \times n$ symmetric positive semidefinite matrix

$$
\mathcal{R}=\mathcal{R}^{\top}=\operatorname{diag}\left\{\left[\begin{array}{ll}
\mathbf{0}_{m}^{\top} & 1 / R
\end{array}\right]\right\} \geq 0,
$$

while the skew-symmetric matrix

$$
\mathcal{J}=-\mathcal{J}^{\top}=\left[\begin{array}{cc}
\mathbf{0} & -\mathbf{1}_{m} \\
\mathbf{1}_{m}^{\top} & 0
\end{array}\right] \in \mathbb{R}^{n \times n},
$$

together with

$$
B=\left[\begin{array}{c}
\operatorname{diag}\{E\} \\
\mathbf{0}_{m}^{\top}
\end{array}\right] \in \mathbb{R}^{n \times m},
$$

represent the interconnection structure. See [5], [12], [13] for more details about PCH modelling.

Bus voltage regulation to a given value $v_{r} \in \mathbb{R}_{>0}$ (or equivalently $\mathbb{R}_{>0} \ni Q_{r}:=C v_{r}$ ) represents the main control objective. We will see later on that the voltage $v$ and hence $Q$ as well only depends on the total current, i.e. the sum of each $i_{k}$. Thus additional degrees of freedom remain in the way this total current is distributed among the converters. Thereby this paper considers current control distribution or, equivalently, flux control distribution as an additional control objective which is independent from voltage regulation. This secondary control objective is conveniently expressed as a cost function to be minimized, corresponding for example to power losses. This leads to the following expression of the problem addressed in this paper: The constraints correspond to the main control objective whereas the optimization form is related to the secondary objective.

Problem 1. Given a cost function $J: \mathbb{R}^{m+1} \rightarrow \mathbb{R}$, design state feedback control law $x \mapsto d$ such that resulting closed-loop system admits a (unique) equilibrium point

$$
x^{\star}:=\left[\begin{array}{c}
\varphi^{\star} \\
Q^{\star}
\end{array}\right]:=\underset{\varphi, Q}{\operatorname{argmin}} J(\varphi, Q) \text { s.t. }\left\{\begin{array}{c}
Q=Q_{r} \\
{\left[\begin{array}{c}
\dot{\varphi} \\
\dot{Q}
\end{array}\right]=\mathbf{0}}
\end{array}\right.
$$

which is globally and asymptotically stable (GAS).

Literature for Problem 1 is well surveyed in [14], [3]. Remarkably, almost all existing solutions make use of a two nested loops scheme. The first loop aims associating a close control to each converter, making them acts as a controlled voltage or current source. The second loop is an outer controller whose goal is to achieve exact voltage and current regulation. The fundamental tool for achieving closed-loop stability is frequency separation between those two loops. However, accelerate the outer loop in order to enhance the voltage dynamics might break the frequency separation which, in turn, leads to instability.

In stark contrast with this approach, strategy proposed in the sequel does not required any frequency separation, by resorting to a peculiar change of coordinates.

\section{Change of COORdinates}

Purpose of current section is to provide both state and input change of coordinates which aim to separate dynamics into: (i) the minimal part of state vector related to voltage dynamics, i.e. total current and voltage and (ii) the remaining dynamics that is current distribution.

\section{A. Separation of dynamics}

From Fig. 1, it is clear that the output voltage $v$ only depends on the sum of currents $i_{k}$ rather than current of each branch individually. The following change of coordinates aims to highlight this observation. Accordingly, we introduce the total flux related to the total current as

$$
\varphi_{T}:=L_{\mathrm{eq}, m} \sum_{k=1}^{m} \frac{\varphi_{k}}{L_{k}}=\mathbf{1}_{m}^{\top} \operatorname{diag}\{L\}^{-1} L_{\mathrm{eq}, m} \varphi
$$

with $L_{\text {eq, } k}$ being the equivalent inductor of the $k$ first coils connected in parallel (see Fig. 2 for $k=m$ ): ${ }^{1}$

$$
\frac{1}{L_{\mathrm{eq}, k}}:=\sum_{j=1}^{k} \frac{1}{L_{j}} \neq 0
$$

We also introduce $\mathcal{C} \in \mathbb{R}^{m-1}$ that reflect flux distribution related to the current distribution as

$$
\mathcal{C}=\Gamma_{m}^{\top} \varphi
$$

with

$$
\mathbb{R}^{(m-1) \times m} \ni \Gamma_{m}^{\top}:=G-\left[\begin{array}{ll}
\mathbf{0}_{m-1} & \mathbf{I}_{m-1}
\end{array}\right],
$$

\footnotetext{
${ }^{1} L_{\text {eq, } m}$ has been introduced in order to make $\varphi_{T}$ homogeneous to magnetic flux.
} 


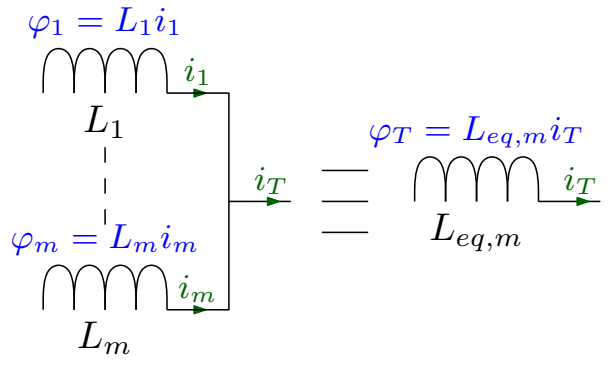

Fig. 2: Physical interpretation of $\varphi_{T}$ where the $k$-th row of $G \in \mathbb{R}^{(m-1) \times m}$ is

$$
\begin{aligned}
& G_{k}:=\left[\begin{array}{ll}
L_{\mathrm{eq}, k} \mathbf{1}_{k}^{\top} & \mathbf{0}_{m-k}^{\top}
\end{array}\right] \operatorname{diag}\{L\}^{-1} \\
& =L_{\mathrm{eq}, k}\left[\begin{array}{lllllll}
\frac{1}{L_{1}} & \frac{1}{L_{2}} & \cdots & \frac{1}{L_{k}} & 0 & \cdots & 0
\end{array}\right] .
\end{aligned}
$$

Note that the remark named Expression of $\Gamma_{m}$ in p.5 gives guidelines for the construction of $\mathcal{C}$ for $m=3$.

Next lemma provides dynamical equations in the new coordinates after introducing new input coordinates.

Lemma 1. Let $\Phi^{-1}$ and $U^{-1}$ define state and input change of coordinates via $z:=\left[\begin{array}{lll}\mathcal{C}^{\top} & \varphi_{T} & Q\end{array}\right]^{\top}=$ $\Phi^{-1} x$ and $\left[\begin{array}{ll}\lambda^{\top} & \mu\end{array}\right]^{\top}=U^{-1} d$ where

$$
\Phi^{-1}=\left[\begin{array}{cc}
\Gamma_{m}^{\top} & \mathbf{0}_{m-1} \\
\mathbf{1}_{m}^{\top} \operatorname{diag}\{L\}^{-1} L_{\mathrm{eq}, m} & 0 \\
\mathbf{0}_{m}^{\top} & 1
\end{array}\right]
$$

and

$$
\begin{aligned}
U:=\operatorname{diag}\{E\}^{-1}\left[\begin{array}{c}
\Gamma_{m}^{\top} \\
\mathbf{1}_{m}^{\mathrm{T}} \operatorname{diag}\{L\}^{-1} \\
L_{\mathrm{eq}, m}
\end{array}\right]^{-1} \\
{\left[\begin{array}{cc}
\operatorname{diag}\{\tilde{E}\} & \mathbf{0} \\
\mathbf{0} & E_{\mathrm{eq}}
\end{array}\right], }
\end{aligned}
$$

with $\tilde{E} \in \mathbb{R}^{m-1}$ and $E_{\text {eq }} \in \mathbb{R}$ being positive parameters to be chosen. Model (2) can be equivalently rewritten as

$$
\begin{aligned}
& \dot{z}= \underbrace{\left[\begin{array}{ccc}
\mathbf{0} & \mathbf{0}_{m-1} & \mathbf{0}_{m-1} \\
\mathbf{0}_{m-1}^{\top} & 0 & -1 \\
\mathbf{0}_{m-1}^{\top}-1 & 1 & -1 / R
\end{array}\right]}_{\mathcal{J}_{z}-\mathcal{R}_{z}} \nabla_{z} H_{z}(z) \\
&+\left[\begin{array}{cc}
\operatorname{diag}\{\tilde{E}\} & \mathbf{0}_{m-1} \\
\mathbf{0}_{m-1}^{\top} & E_{\text {eq }} \\
\mathbf{0}_{m-1}^{\top} & 0
\end{array}\right]\left[\begin{array}{c}
\lambda \\
\mu
\end{array}\right] .
\end{aligned}
$$

where $H_{z}(z)=\frac{1}{2} z^{\top} \mathcal{Q} z$ with $\mathcal{Q}$ being the following positive definite diagonal matrix

$$
\mathcal{Q}:=\operatorname{diag}\left\{L_{\mathcal{C}}, L_{\mathrm{eq}, m}, C\right\}^{-1}
$$

with $L_{\mathcal{C}}$ a $(m-1)$-dimensional vector defined by

$$
L_{\mathcal{C}, k}:=L_{\mathrm{eq}, k}+L_{k+1}, \quad \forall k \in\{1, \cdots, m-1\} .
$$

Proof: Since $\Gamma_{m}^{\top}$ is full-row rank, $\mathbf{1}_{m}^{\top} \operatorname{diag}\{L\}^{-1} \mathbf{1}_{m}=1 / L_{\mathrm{eq}, m}$ and $\Gamma_{m}^{\top} \mathbf{1}_{m}=\mathbf{0}_{m-1}$, we can show that $\Phi$ reads

$$
\Phi=\left[\begin{array}{ccc}
\Gamma_{m}^{+} & \mathbf{1}_{m} & \mathbf{0}_{m} \\
\mathbf{0}_{m-1}^{\top} & 0 & 1
\end{array}\right],
$$

where

$$
\Gamma_{m}^{+}:=\frac{\operatorname{diag}\{L\}}{L_{\mathrm{eq}, m}} \Gamma_{m}\left(\Gamma_{m}^{\top} \frac{\operatorname{diag}\{L\}}{L_{\mathrm{eq}, m}} \Gamma_{m}\right)^{-1} .
$$

System in hamiltonian form reads

$$
\begin{aligned}
\dot{z}=\Phi^{-1} \dot{x}=\underbrace{\Phi^{-1}[\mathcal{J}-\mathcal{R}] \Phi^{-\top}}_{=: \mathcal{J}_{z}-\mathcal{R}_{z}} \nabla_{z} H_{z}(z) \\
+\Phi^{-1}\left[\begin{array}{c}
\operatorname{diag}\{E\} \\
\mathbf{0}_{m}^{\top}
\end{array}\right] d
\end{aligned}
$$

where the Hamiltonian is such that

$$
H_{z}(z)=H(\Phi(z))=\frac{1}{2} z^{\top} \mathcal{Q} z
$$

with $\mathcal{Q}=\mathcal{Q}^{\top}>0$ being the following block-diagonal matrix

$$
\mathcal{Q}=\left[\begin{array}{cc}
\left(\Gamma_{m}^{+}\right)^{\top} \operatorname{diag}\{L\}^{-1} \Gamma_{m}^{+} & \mathbf{0}_{m-1} \\
\mathbf{0}_{m-1}^{\top} & \operatorname{diag}\left\{L_{\mathrm{eq}, m}, C\right\}^{-1}
\end{array}\right] .
$$

Using (7), it holds $\left(\Gamma_{m}^{\top} \operatorname{diag}\{L\} \Gamma_{m}\right)^{-\top}=:\left(\left[a_{i j}\right]\right)^{-1}$ with

$$
\begin{aligned}
a_{i i} & =\left(L_{\mathrm{eq}, i}\right)^{2} \underbrace{\left(\sum_{k=1}^{i} \frac{1}{L_{k}}\right)}_{L_{\mathrm{eq}, i}^{-1}}+L_{i+1}=L_{\mathcal{C}, i} \\
i<j, a_{i j} & =L_{\mathrm{eq}, i} L_{\mathrm{eq}, j} \underbrace{\left(\sum_{k=1}^{i} \frac{1}{L_{k}}\right)}_{L_{\mathrm{eq}, i}^{-1}}-L_{\mathrm{eq}, j}=0 .
\end{aligned}
$$

Thus $\left(\Gamma_{m}^{+}\right)^{\top} \operatorname{diag}\{L\}^{-1} \Gamma_{m}^{+}=\operatorname{diag}\left\{L_{\mathcal{C}}\right\}^{-1}$ so that (10) holds. Furthermore, $\mathcal{J}_{z}-\mathcal{R}_{z}$ appearing in (12) reduces to

$$
\begin{aligned}
\mathcal{J}_{z}-\mathcal{R}_{z} & =\Phi^{-1}[\mathcal{J}-\mathcal{R}] \Phi^{-\top} \\
& =\left[\begin{array}{ccc}
\mathbf{0} & \mathbf{0}_{m-1} & \mathbf{0}_{m-1} \\
\mathbf{0}_{m-1}^{\top} & 0 & -1 \\
\mathbf{0}_{m-1}^{\top} & 1 & -1 / R
\end{array}\right] .
\end{aligned}
$$

Finally, input to state matrix reads

$$
\Phi^{-1}\left[\begin{array}{c}
\operatorname{diag}\{E\} \\
\mathbf{0}_{m}^{\top}
\end{array}\right] U=\left[\begin{array}{c}
\operatorname{diag}\left\{\tilde{E}, E_{\mathrm{eq}}\right\} \\
\mathbf{0}_{m}^{\top}
\end{array}\right] .
$$



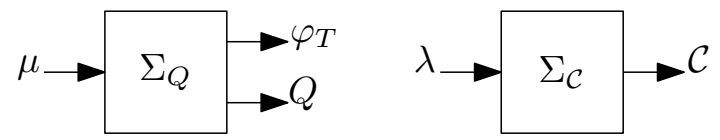

Fig. 3: New open-loop model.

Remark (Input change of coordinates). After the state transformation that separate flux distribution from voltage and total current dynamics, the input change of coordinates aims to find the input part that only acts on $\mathcal{C}$ and the one that only acts on $\varphi_{T}$ and $Q$.

From sparsity of matrices in (9), we are able to separate (9) into two independent subsystems:

- The first one, $\Sigma_{\mathcal{C}}$ is described by the $(m-1)$ first lines of (9):

$$
\begin{cases}\dot{\mathcal{C}} & =\operatorname{diag}\{\tilde{E}\} \lambda \\ H_{\mathcal{C}}(\mathcal{C}) & =\frac{1}{2} \mathcal{C}^{\top} \operatorname{diag}\left\{L_{\mathcal{C}}\right\}^{-1} \mathcal{C}\end{cases}
$$

and corresponds to the dynamics of overall flux repartition among the different branches;

- The second one, $\Sigma_{Q}$ is described by the two last lines of (9):

$$
\left\{\begin{array}{l}
{\left[\begin{array}{c}
\dot{\varphi_{T}} \\
\dot{Q}
\end{array}\right]=\underbrace{\left[\begin{array}{cc}
0 & -1 \\
1 & -1 / R
\end{array}\right]}_{\mathcal{J}_{Q}^{-\mathcal{R}_{Q}}} \nabla_{\varphi_{T}, Q} H_{Q}+\left[\begin{array}{c}
E_{\mathrm{eq}} \\
0
\end{array}\right] \mu} \\
H_{Q}=\frac{1}{2}\left[\begin{array}{c}
\varphi_{T} \\
Q
\end{array}\right]^{\top} \operatorname{diag}\left\{L_{\mathrm{eq}, m}, C\right\}^{-1}\left[\begin{array}{c}
\varphi_{T} \\
Q
\end{array}\right],
\end{array}\right.
$$

and governs dynamics of the capacitor charge, through equivalent flux $\varphi_{T}$ controlled by input $\mu$.

This separation is represented on Fig. 3.

Remark (Fully disconnected subsystems). Vis-avis the main result of [4] where the change of coordinates leads to cascaded subsystems, the Hamiltonian formulation gives rise to a different change of coordinates that fully decouples the two dynamics $\Sigma_{\mathcal{C}}$ and $\Sigma_{Q}$. Furthermore it induces a diagonal structure of $\Sigma_{\mathcal{C}}$, dynamics of $\mathcal{C}$ as well as $H_{\mathcal{C}}$.

Remark (Expression of $\Gamma_{m}$ ). Note that $\Gamma_{m}$ could have been defined differently while preserving this aforementioned separation between dynamics. However, we will see in the following that this particular expression leads to a nice structure of the system in the new coordinates and a physical interpretation. Indeed, take the equivalent inductor of the $k$ first branches and the sum of the $k$ first currents, their

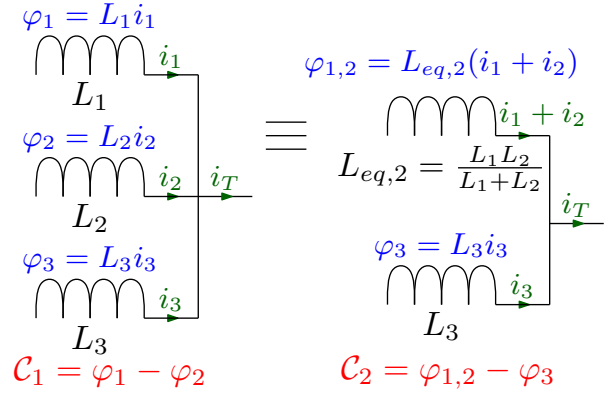

Fig. 4: Physical representation of $\mathcal{C}$.

product corresponds to $G_{k} x$, the equivalent flux of the $k$ first inductors (see Fig. 4). As an example, for $k=2, G_{2} x$ reads

$$
G_{2} x=\frac{1}{1 / L_{1}+1 / L_{2}}\left(\frac{\varphi_{1}}{L_{1}}+\frac{\varphi_{2}}{L_{2}}\right)=L_{\mathrm{eq}, 2}\left(i_{1}+i_{2}\right)
$$

\section{B. Circuit theory interpretation}

On the one hand, from its dynamical equation (14), subsystem $\Sigma_{Q}$ can be physically interpreted as average model of buck converter depicted on Fig. 5 (a) (see [6]). The equivalent inductor $L_{\mathrm{eq}, m}$ of $m$ coils in parallel and the equivalent source $E_{\text {eq }}$ compose the converter, whereas the input $\mu$ act as a virtual duty cycle. The converter feeds the load $R$ through the capacitor $C$.

On the other hand, $\Sigma_{\mathcal{C}}$ can be seen as $(m-1)$ independent electrical circuits $\Sigma_{\mathcal{C}}^{k}$ represented on Fig. 5 (b). Each electrical circuit is composed by an electrical source $\tilde{E}_{k}$ connected to a virtual coil $L_{\mathcal{C}, k}$ via controllable transistors. The circuit is passed through by the following electrical flow $\mathcal{C}_{k}=l_{\mathcal{C}, k} \bar{i}_{k}$ where $\tilde{i}_{k}=\frac{L_{\mathrm{eq}, k}}{L_{\mathcal{C}, k}}\left(\frac{\varphi_{1}}{L_{1}}+\cdots+\frac{\varphi_{k}}{L_{k}}-\frac{\varphi_{k+1}}{L_{\mathrm{eq}, k}}\right)$. By the virtual duty cycle $\lambda_{k}$, we can control the electrical flow $\mathcal{C}_{k}$. If $\mathcal{C}_{k}$ is positive, the $k$ first branches will be favoured to transmit power to the load whereas with $\mathcal{C}_{k}<0$, the branch $k+1$ will take more power than the $k$ first ones. Because subsystems $\Sigma_{\mathcal{C}}^{k}$ are independent from the equivalent buck converter, $\mathcal{C}_{k}$ are only specifying how power flow is allocated among the branches without affecting the power transmitted to the load. Fig. 5 (b) depicts that $\Sigma_{\mathcal{C}}^{k}$ corresponds to the energy transiting between the equivalent inductance $L_{\mathrm{eq}, k}$ and $L_{k+1}$ (because $L_{\mathcal{C}, k}=L_{\mathrm{eq}, k}+L_{k+1}$ ).

\section{Reformulation of Problem 1 in the new coordi- nates}

So far, the contribution is to separate what acts on the charge $Q$, that is $\varphi_{T}$ and $\mu$ from the free 


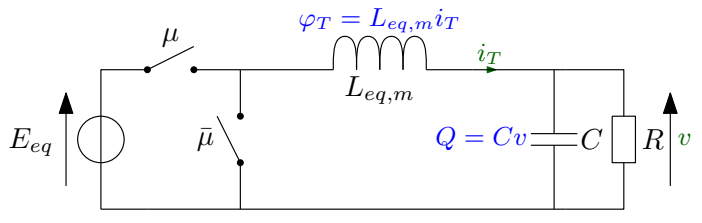

(a)

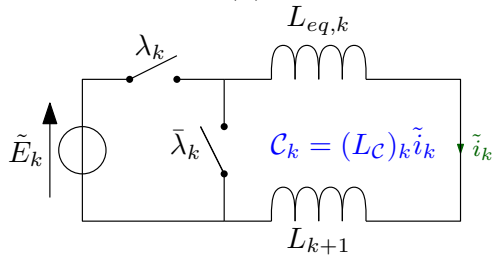

(b)

Fig. 5: Circuit interpretation of (a) $\Sigma_{v}$ and (b) $\Sigma_{\mathcal{C}}^{k}$.

variables $\mathcal{C}$ and inputs $\lambda$. Let us rewrite the equation of Problem 1 in the variables defined in the previous section:

$$
\begin{aligned}
{\left[\mathcal{C}^{* \top}, \varphi_{T}^{\star}, Q^{\star}\right]^{\top}:=\underset{\mathcal{C}, \varphi_{T}, Q}{\operatorname{argmin}} } & J(\Phi z) \\
\text { s.t. } & \left\{\begin{array}{l}
Q=Q_{r} \\
\dot{z}=\mathbf{0}_{m+1} .
\end{array}\right.
\end{aligned}
$$

Knowing the constraint about equilibrium point $\left(Q=Q_{r}\right)$, it follows from (1b) that

$$
\sum_{k=1}^{m} \frac{\varphi_{k}^{\star}}{L_{k}}=\frac{Q_{r}}{R C} \Leftrightarrow \varphi_{T}^{\star}=\frac{L_{\mathrm{eq}, m} Q_{r}}{R C} .
$$

Thus, the constraints impose the asymptotic values of variables related to the equivalent buck (i.e. $\varphi_{T}$ and $Q$ ) and they are no longer decision variable of the optimization problem. Hence, optimization subproblem of Problem 1 reduces to:

$$
\mathcal{C}^{\star}:=\underset{\mathcal{C}}{\operatorname{argmin}} J_{z}\left(\mathcal{C}, \varphi_{T}^{\star}\right) \text { s.t. } \dot{\mathcal{C}}=\mathbf{0}_{m-1},
$$

where $J_{z}$ and $\varphi_{T}^{\star}$ read

$$
\begin{aligned}
J_{z} & :\left(\mathcal{C}, \varphi_{T}\right) \mapsto J\left(\Phi\left[\begin{array}{lll}
\mathcal{C}^{\top} & \varphi_{T} & Q_{r}
\end{array}\right]^{\top}\right) \\
\varphi_{T}^{\star} & :=L_{\text {eq }, m} Q_{r} /(R C)
\end{aligned}
$$

The separation in two blocs confines dynamics of $\varphi_{T}$ and $Q$ in a single subsystem. Independently of cost function $J_{z}$, those two variables must converges to $\varphi_{T}^{\star}$ and $Q_{r}$ respectively to solve Problem 1 . In fact, this dynamics refers to voltage regulation objective. Second subsystem $\Sigma_{\mathcal{C}}$ of variables $\mathcal{C}$ must converge to an optimal value of cost function $J_{z}$. This dynamics refers to the optimization of power flow repartition among all the converters, for a chosen total power transmitted to the load.
Remark (Casimir functions). In this section, we gave a change of coordinates that decompose the system in two parts to separate voltage regulation from flow distribution. In fact, this change of coordinates is closely related to the presence of Casimir functions. A Casimir function $\mathcal{C}: \mathbb{R}^{n} \rightarrow \mathbb{R}$ is a conservative function regardless of the Hamiltonian (see [12, p.87]). It expresses the dynamical invariants and is a solution of

$$
\frac{\mathrm{d} \mathcal{C}}{\mathrm{d} t}=0 \Leftrightarrow \frac{\partial \mathcal{C}}{\partial x} \dot{x}=0 .
$$

As the property of $\mathcal{C}$ holds for all $H$, by including the model of an autonomous Hamiltonian system into (16) we obtain the following relation

$$
\frac{\partial \mathcal{C}}{\partial x}(x)[\mathcal{J}(x)-\mathcal{R}(x)]=0 .
$$

From [12, p 87], we know that Casimir functions can be used in order to achieve a change of coordinates that isolate those functions from the rest of the state. Since $\Gamma_{m}^{\boldsymbol{T}} \mathbf{1}_{m}=\mathbf{0}$ and

$$
\frac{\partial \mathcal{C}}{\partial x}=\left[\begin{array}{ll}
\Gamma_{m}^{\top} & \mathbf{0}_{m}
\end{array}\right]
$$

with $\mathcal{C}$ given in (6), it is clear that (17) is satisfied, so that every entry of vector $\mathcal{C}$ is a Casimir function.

\section{Control DESIGN}

Starting from the case where the load is known (Subsection IV-A), this section then gives a loadindependent solution to Problem 1 (see Subsection IV-B).

\section{A. Control design with known $R$}

Firstly, we want to solve Problem 1 when $R$ is known.

1) Control of equivalent Buck $\Sigma_{Q}$ : Let us first design a controller for $\Sigma_{Q}$ that ensure that $Q \rightarrow$ $Q^{\star}=Q_{r}$ and $\varphi_{T} \rightarrow \varphi_{T}^{\star}$. Here, the controller is based on the well known Interconnection and Damping Assignment Passivity-Based Control (IDA-PBC) procedure introduced in [8]. The desired closed-loop behaviour is written as the following Hamiltonian system

$$
\left[\begin{array}{c}
\dot{\varphi}_{T} \\
\dot{Q}
\end{array}\right]=\left[\mathcal{J}_{Q}^{d}-\mathcal{R}_{Q}^{d}\right] \nabla_{\varphi_{T}, Q} H_{Q}^{d}\left(\varphi_{T}, Q\right),
$$

where

$$
\begin{aligned}
\mathcal{J}_{Q}^{d} & :=\left[\begin{array}{cc}
0 & -1 \\
1 & 0
\end{array}\right], \quad \mathcal{R}_{Q}^{d}:=\left[\begin{array}{cc}
k_{\mu} & 0 \\
0 & 1 / R
\end{array}\right], \\
H_{Q}^{d} & :=\frac{1}{2 L_{\mathrm{eq}, m}}\left(\varphi_{T}-\frac{L_{\mathrm{eq}, m} Q_{r}}{R C}\right)^{2}+\frac{1}{2 C}\left(Q-Q_{r}\right)^{2} .
\end{aligned}
$$


Minimum of $H_{Q}^{d}$ is reached for $Q=Q_{r}$ and $\varphi_{T}=\varphi_{T}^{\star}$. To obtain this closed-loop, we apply the following state feedback controller

$$
\mu=-\frac{k_{\mu}}{E_{\mathrm{eq}}} \frac{1}{L_{\mathrm{eq}, m}}\left(\varphi_{T}-\frac{L_{\mathrm{eq}, m} Q_{r}}{R C}\right)+\frac{Q_{r}}{C} .
$$

Proposition 1. Equilibrium point $\left(\varphi_{T}^{\star}, Q_{r}\right)$ of closed-loop (18) is GAS for any $R>0$ if $k_{\mu}>0$.

Proof: $H_{Q}^{d}$ is a strictly convex function (quadratic) which is minimum at $\left(\varphi_{T}^{\star}, Q_{r}\right)$. Furthermore, $k_{\mu}>0$ and $R>0$ ensure $\mathcal{R}_{d}>0$, such that

$$
\forall \varphi_{T}, Q, \quad \dot{H}_{d}=-\left(\nabla_{\varphi_{T}, Q} H_{Q}^{d}\right)^{\top} \mathcal{R}_{d} \nabla_{\varphi_{T}, Q} H_{Q}^{d}<0 .
$$

Thus $H_{d}$ is a Lyapunov function of (18) and the closed-loop converges globally and asymptotically to the state value where $H_{d}$ is minimum, that is $\left(\varphi_{T}^{\star}, Q_{r}\right)$

2) Control of $\Sigma_{\mathcal{C}}$ : Let us define the control law

$$
\lambda=-\operatorname{diag}\{\tilde{E}\}^{-1} K_{\lambda} \nabla_{\mathcal{C}} J_{z}\left(\mathcal{C}, \varphi_{T}^{\star}\right),
$$

where $K_{\lambda}=K_{\lambda}^{\top} \in \mathbb{R}^{(m-1) \times(m-1)}$. In such a case, the closed-loop dynamics reads

$$
\dot{\mathcal{C}}=-K_{\lambda} \nabla_{\mathcal{C}} J_{z}\left(\mathcal{C}, \varphi_{T}^{\star}\right) .
$$

Assumption 1. Map $J_{z}$ is (i) continuously differentiable and such that (ii) for all $\varphi_{T}>0$, map $J_{z}\left(\cdot, \varphi_{T}\right)$ is strictly convex ${ }^{2}$ and admits a minimum.

Proposition 2. If Assumption 1 hold and $K_{\lambda}$ is a positive definite matrix, then the closed-loop (21) converges globally and asymptotically to the value where the cost function $J_{z}\left(\cdot, \varphi_{T}^{\star}\right)$ is minimum.

Proof: By convexity, minimum of $J_{z}\left(\cdot, \varphi_{T}\right)$ is unique for all $R>0$. Indeed, assumption on $J_{z}\left(\cdot, \varphi_{T}\right)$ (existence of minimum and strict convexity) applies for all $\varphi_{T}>0$ and, in turn, for all $\varphi_{T}^{\star}(R)$ as soon as $R>0$ (see definition of $\varphi_{T}^{\star}(R)$ ). As

$$
\dot{J}_{z}\left(\mathcal{C}, \varphi_{T}^{\star}\right)=-\left(\nabla_{\mathcal{C}} J_{z}\right)^{\top} K_{\lambda} \nabla_{\mathcal{C}} J_{z}<0
$$

if $K_{\lambda}>0, J_{z}\left(\mathcal{C}, \varphi_{T}^{\star}\right)$ is a Lyapunov function for the closed-loop (21). The state value where $J_{z}\left(\mathcal{C}, \varphi_{T}^{\star}\right)$ is minimum is then a GAS equilibrium point of this closed-loop.

\footnotetext{
${ }^{2}$ Note that for the experimentations described in Section VI, $J$ reflects the converter losses. Relevant from an engineering view point, this cost function is strictly convex.
}

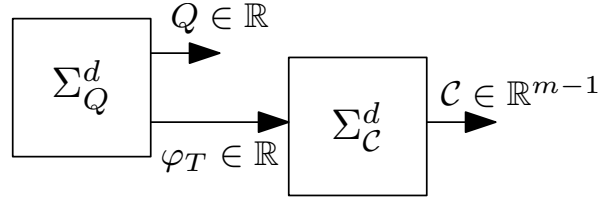

Fig. 6: Cascaded interconnection

3) Solution of Problem 1: Finally, resulting from control of both $\Sigma_{Q}$ and $\Sigma_{\mathcal{C}}$, we enunciate the following theorem:

Theorem 1. If $k_{\mu}>0, K_{\lambda} \succ 0$ and Assumption 1 hold, then load dependent control law defined by (19), (20) and $d=U\left[\begin{array}{ll}\lambda^{\top} & \mu\end{array}\right]^{\top}$ with $U$ giving by (8) solves Problem 1 for all $R>0$.

Proof: From Proposition 1, we know that the constraint $Q=Q_{r}$ of Problem 1 is fulfilled. As explained in Section III-C, set of decision variables of optimization problem of Problem 1 reduces to $\mathcal{C}$. Since controller $(20)$ ensure that $J_{z}\left(\mathcal{C}, \varphi_{T}^{\star}\right)$ is minimum with respect to $\mathcal{C}$ (see Proposition 2), then Problem 1 is solved.

\section{B. Control design with unknown $R$}

Considering that $R$ is unknown leads to two problems:

- For the control of $\Sigma_{Q}$, the previous section shows that a classical IDA-PBC controller requires the load value (eq. (19) relies on $R$ ) because steadystate value depends on $R$;

- The cost function $J$ potentially depends on $\varphi_{T}^{\star}$, the equilibrium value of $\varphi_{T}$ and therefore on $R$. As the controller of $\Sigma_{\mathcal{C}}$ relies on the gradient of $J\left(\mathcal{C}, \varphi_{T}^{\star}\right)$, it also depends on $R$.

One of the consequences is that a unilateral interconnection (variable $\varphi_{T}$ ) is introduced to estimate the load magnitude for each controllers. Hence, the desired closed loop model shall adopt cascaded form depicted on Fig. 6 where $\Sigma_{Q}^{d}$ and $\Sigma_{\mathcal{C}}^{d}$ refer to desired closed loop of $\Sigma_{Q}$ and $\Sigma_{\mathcal{C}}$.

One way to deal with this cascaded form is to use main result of [15]. It comes out that if the upper subsystem (for us $\Sigma_{Q}^{d}$ ) has a GAS equilibrium and if the lower subsystem (for us $\Sigma_{\mathcal{C}}^{d}$ ) has a 0-GAS equilibrium (i.e. GAS when the input is identically 0 ), it simply requires that trajectories are bounded for the equilibrium of the entire system to be GAS.

1) PID-like control of equivalent Buck $\Sigma_{Q}$ : In this subsection, we are interested in controlling the equivalent Buck converter (14) by getting rid of the load value dependance. Robust energy shaping control 
methods (see [9]) take into account this kind of issues. They consist in interconnecting the system with a $\mathrm{PCH}$ controller. This controller acts as an integrator, hence it is also named PI-like control.

In our case, the regulated output (state $Q$ ) does not corresponds to the passive output of the $\mathrm{PCH}$ system (14) (state $\left.\varphi_{T}\right)$. As a result, with a classical interconnection to an integral controller, we are not able to design a $R$-independent control law that stabilize the system at $Q^{\star}=Q_{r}$. Hence we resort to the interconnection of $\mathrm{PCH}$ systems on non-passive outputs. In [10], an approach is presented in order to deal with the case of integral control on non-passive outputs in the Hamiltonian form.

Remark (Unknown parameter). Dealing with unknown parameter $R$ can be cast into a problem of disturbance rejection by considering that deviation of $R$ with respect to its nominal value $R_{0}$ is a perturbation to be rejected by the closed-loop system. Letting $\Delta G$ be defined by $1 / R=1 / R_{0}+\Delta G$, matrices $\mathcal{J}_{Q}-\mathcal{R}_{Q}$ of system (14) reads

$$
\mathcal{J}_{Q}-\mathcal{R}_{Q}=\left[\begin{array}{cc}
0 & -1 \\
1 & -\left(1 / R_{0}+\Delta G\right)
\end{array}\right]
$$

As a result, and in stark contrast with methodology proposed in [10] which focuses on constant exogeneous disturbance, we have to tackle disturbance $\Delta G$ which is multiplied by $\nabla H_{Q}$ in the expression of derivative of $\left(\varphi_{T}, Q\right)$ and is, in turn, (linearly) state-dependent. Yet results of [10] paves the way in construction of robust control law which is proposed in this paper.

Let $C_{\mu}$ be the control law

$$
\left\{\begin{array}{l}
\dot{\xi}=k_{i} \frac{Q-Q_{r}}{C} \\
\mu=-\frac{1}{E_{\mathrm{eq}}}\left(k_{d} \frac{\varphi_{T}}{L_{\mathrm{eq}, m}}+k_{d} \xi+L_{\mathrm{eq}, m} k_{i} \frac{Q-Q_{r}}{C}\right)
\end{array}\right.
$$

with $k_{d}, k_{i} \in \mathbb{R}$ be the integral controller on the nonpassive output of system (14).

Proposition 3. The closed-loop of (14) and (22) converges globally and asymptotically to some equilibrium point for which $Q$ equals $Q_{r}$ for all $R>0$ if $k_{d}>0$ and $k_{i}>0$.

Proof: From [10] one can prove that the integral controller (22) and system (14) can be written in the following coordinates

$$
\chi:=\left[\begin{array}{ccc}
1 & 0 & L_{\mathrm{eq}, m} \\
0 & 1 & 0 \\
0 & 0 & 1
\end{array}\right]\left[\begin{array}{c}
\varphi_{T}-\frac{L_{\mathrm{eq}, m}}{R C} Q_{r} \\
Q-Q_{r} \\
\xi-\left(-\frac{1}{k_{d}}-\frac{1}{R}\right) \frac{Q_{r}}{C}
\end{array}\right]
$$

as the Hamiltonian system:

$$
\dot{\chi}=\left[\begin{array}{ccc}
-k_{d} & -1 & 0 \\
1 & -1 / R & -k_{i} \\
0 & k_{i} & 0
\end{array}\right] \nabla_{\chi} H_{d}(\chi)
$$

where

$$
H_{d}(\chi)=\frac{1}{2} \chi^{\top} \operatorname{diag}\left\{\left[L_{\mathrm{eq}, m}, C, k_{i}\right]\right\}^{-1} \chi .
$$

By specifying $k_{i}>0$, we ensure that (25) is a strictly convex function over $\mathbb{R}^{3}$. Furthermore, it directly follows from (25) that it admits a (unique) minimum at $\chi^{\star}=\mathbf{0}_{3}$. In addition to that, $\dot{H}_{d}=\left(\nabla_{\chi} H_{d}(\chi)\right)^{\top} \mathcal{R}_{d} \nabla_{\chi} H_{d}(\chi) \leq 0$ with $\mathcal{R}_{d}:=\operatorname{diag}\left\{\left[k_{d}, 1 / R, 0\right]\right\}$. Knowing that the largest invariant contained in

$$
\mathcal{S}:=\left\{\chi \in \mathbb{R}^{3} \mid \dot{H}_{d}=0\right\}=\left\{\chi \in \mathbb{R}^{3} \mid \chi_{1}=\chi_{2}=0\right\}
$$

is $\left\{\mathbf{0}_{3}\right\}$ because

$$
\begin{array}{r}
\dot{\chi}=\left[\begin{array}{ccc}
-k_{d} & -1 & 0 \\
1 & -1 / R & -k_{i} \\
0 & k_{i} & 0
\end{array}\right] \nabla_{\chi} H_{d}\left(0,0, \chi_{3}\right) \subseteq \mathcal{S} \\
\Leftrightarrow\left(0,-k_{i} \chi_{3}, 0\right) \subseteq \mathcal{S} \quad \Leftrightarrow \quad \chi_{3}=0,
\end{array}
$$

it follows from LaSalle's Invariance Principle (see [16]) that $\chi^{\star}=\mathbf{0}_{3}$ is GAS. Furthermore, it is clear from (23) that when $\chi=\mathbf{0}_{3}$, it holds $Q=Q_{r}$.

Remark (Controller properties). Gain $k_{d}$ refers to the feedback of the flux variable $\varphi_{T}$ whereas $k_{i}$ have an influence on the integral action of the charge $Q$. We also notice that: (i) if $k_{d}=0$, then there is no integral action of the controller and (ii) $k_{i}$ also have a proportional feedback action on the charge $Q$.

2) Control of $\Sigma_{\mathcal{C}}$ : In general, the cost function $J_{z}\left(\cdot, \varphi_{T}^{\star}\right)$ depends on the load $R$ via $\varphi_{T}^{\star}$ which is unknown. This is the reason why, we consider the following control law of $\Sigma_{\mathcal{C}}$, in place of (20),

$$
C_{\lambda}: \quad \lambda=-\operatorname{diag}\{\tilde{E}\}^{-1} K_{\lambda} \nabla_{\mathcal{C}} J_{z}\left(\mathcal{C}, \varphi_{T}\right)
$$

which leads to the closed loop

$$
\Sigma_{\mathcal{C}}^{d}: \quad \dot{\mathcal{C}}=-K_{\lambda} \nabla_{\mathcal{C}} J_{z}\left(\mathcal{C}, \varphi_{T}\right) .
$$

As already discussed at the beginning of Subsection IV-B, $\varphi_{T}$ is now an input of $\Sigma_{\mathcal{C}}^{d}$ and we will 
see here stability property of this subsystem when this input is at rest, that is when $\varphi_{T}=\varphi_{T}^{\star}$. In such a case, we recover subsystem already considered in Proposition 2, so that the following result can be established in the same way, since $\varphi_{T}^{\star}>0$ is arbitrary in Proposition 2.

Proposition 4. Assume $K_{\lambda} \succ 0$ and Assumption 1 holds. Then for any strictly positive and constant $\varphi_{T}$, set point $\arg \min _{\mathcal{C}} J_{z}\left(\mathcal{C}, \varphi_{T}\right)$ is GAS.

3) Solution of Problem 1: Making use of individual controllers of both $\Sigma_{Q}$ and $\Sigma_{\mathcal{C}}$, we now establish main result of this paper.

Theorem 2. Let $\mathcal{C}^{\star}\left(\varphi_{T}\right):=\arg \min _{\mathcal{C}} J_{z}\left(\mathcal{C}, \varphi_{T}\right)$ and define maps $h: \mathbb{R}^{m-1} \times \mathbb{R} \rightarrow \mathbb{R}^{m-1}$ and $W: \mathbb{R}^{m-1} \rightarrow$ $\mathbb{R}$ as follows:

$$
\begin{array}{r}
h\left(\tilde{\mathcal{C}}, \tilde{\varphi}_{T}\right):=\nabla_{\mathcal{C}} J_{z}\left(\tilde{\mathcal{C}}+\mathcal{C}^{\star}, \tilde{\varphi}_{T}+\varphi_{T}^{\star}\right) \\
-\nabla_{\mathcal{C}} J_{z}\left(\tilde{\mathcal{C}}+\mathcal{C}^{\star}, \varphi_{T}^{\star}\right) \\
W(\tilde{\mathcal{C}}):=J_{z}\left(\tilde{\mathcal{C}}+\mathcal{C}^{\star}, \varphi_{T}^{\star}\right)-J_{z}\left(\mathcal{C}^{\star}, \varphi_{T}^{\star}\right) .
\end{array}
$$

Assume that the two following facts hold:

F1) There exists two strictly increasing functions $G_{1,2}$ which are null and differentiable at the origin and such that $\left\|h\left(\tilde{\mathcal{C}}, \tilde{\varphi}_{T}\right)\right\| \leq G_{1}\left(\left|\tilde{\varphi}_{T}\right|\right)+$ $G_{2}\left(\left|\tilde{\varphi}_{T}\right|\right)\|\tilde{\mathcal{C}}\|$

F2) There exist positive constants $c$ and $k$ such that $\|\tilde{\mathcal{C}}\|>c$ implies $\|\nabla W(\tilde{\mathcal{C}})\|\|\tilde{\mathcal{C}}\| \leq k W(\tilde{\mathcal{C}})$

Then, load independent control law defined by (22), (26) and $d=U\left[\begin{array}{ll}\lambda^{\top} & \mu\end{array}\right]^{\top}$ with $U$ given by (8) solves Problem 1 if $K_{\lambda}=K_{\lambda}^{\top} \succ 0, k_{d}>0, k_{i}>0$ and Assumption 1 holds.

Proof: From Proposition 3, we know that for any $R>0$, there exists $\xi^{\star}(R)$ such that $\left(\varphi_{T}^{\star}(R), Q_{r}, \xi^{\star}(R)\right)$ is an GAS equilibrium for $\Sigma_{Q}^{d}$. From Proposition 4, we have that $\mathcal{C}^{\star}\left(\varphi_{T}\right)=$ $\arg \min _{\mathcal{C}} J_{z}\left(\mathcal{C}, \varphi_{T}\right)$ is a GAS equilibrium of $\Sigma_{\mathcal{C}}^{d}$ for any constant $\varphi_{T}>0$. From [15], in such a case, boundedness of trajectories implies global asymptotic stability of $\left(\mathcal{C}^{\star}\left(\varphi_{T}^{\star}(R)\right), \varphi_{T}^{\star}(R), Q_{r}, \xi^{\star}(R)\right)$ for the whole closed loop system for all $R>0$. To prove boundedness property, first note that existence of a GAS equilibrium for $\Sigma_{Q}^{d}$ implies boundedness of the $\left(\varphi_{T}, Q, \xi\right)$ substate. Then, observe that dynamics of $\Sigma_{\mathcal{C}}^{d}$ can be reformulated as follows using relative coordinates $\tilde{\varphi}_{T}=\varphi_{T}-\varphi_{T}^{\star}$ and $\tilde{\mathcal{C}}=\mathcal{C}-\mathcal{C}^{\star}$ :

$$
\dot{\tilde{\mathcal{C}}}=-K_{\lambda} \nabla_{\mathcal{C}} J_{z}\left(\tilde{\mathcal{C}}+\mathcal{C}^{\star}, \varphi_{T}^{\star}\right)-K_{\lambda} h\left(\tilde{\mathcal{C}}, \tilde{\varphi}_{T}\right) .
$$

In such case, and whenever Assumption 1, F1) and F2) hold, all the hypothesis for the applicability of [17, Lemma 1] are satisfied which proves that

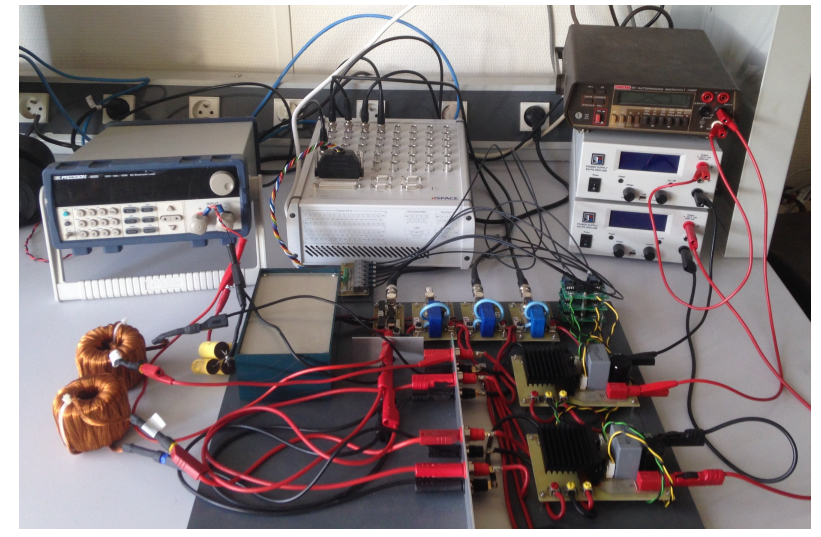

Fig. 7: Experimental test-bench

$W(\mathcal{C}(t))$ remains bounded which, in turn, proves boundedness of $\mathcal{C}(t)$ since $\mathcal{C} \mapsto J_{z}\left(\mathcal{C}, \varphi_{T}\right)$ is radially unbounded for all $\varphi_{T}>0$, due to its convexity and the existence of a minimum.

Remark (About F1) and F2)). Hypothesis F1) imposes linear growth with respect to $\mathcal{C}$ of $h$, the coupling term from $\Sigma_{Q}^{d}$ to $\Sigma_{\mathcal{C}}^{d}$. This prevents finite time escape of $\mathcal{C}$ due to this interaction between subsystems [17]. Regarding F2), [17, Lemma 2] implies that this fact is satisfied if $J_{z}\left(\cdot, \varphi_{T}^{\star}\right)$ is polynomial for any $\varphi_{T}^{\star}>0$, in addition of fulfilling requirements of Assumption 1.

\section{EXPERIMENTATIONS}

In this section, the implementation of the proposed approach is illustrated by experimental results.

The experimental setup, depicted by Fig.7, is composed of 2 heterogeneous buck converters $(m=2)$ in the sense that inductors, as well as transistors are different. The second converter is designed in such a way that its passive elements have lower quality but the switches have a better efficiency. This means that for low power, the use of converter 2 is preferable whereas converter 1 should have priority for high power. See [18] for detailed discussion on this feature.

The controller hardware is a dSpace MicroLabBox. For any $R$, control objectives are (i) regulate charge $Q$ at the reference $Q_{r}=264 \mathrm{mC}$ which corresponds to a voltage reference $v_{r}=12 \mathrm{~V}$ and (ii) impose optimal flux distribution through the converters with respect to a cost function $J$. The load variations are performed by a DC electronic load BK Precision 8600 series with a maximum power of $150 \mathrm{~W}$ and controlled by the dSpace board.

Bench parameters are the followings. The two input voltages are such that $E_{1}=E_{2}=24 \mathrm{~V}$. 
The switching frequency is chosen as $f_{s}=20 \mathrm{kHz}$ whereas the sampling frequency is $f_{e}=10 \mathrm{kHz}$. All the transistors are MOSFET. For the first converter, their references are STP31510F7 and for the second, STP30NF10. Inductor of the first converter is $K_{1}=2.83 \mathrm{mH}$ and for the second is $L_{2}=1.3 \mathrm{mH}$. Finally the output capacitor value is $C=22 \mathrm{mF}$.

For both experiments, we apply the control law (22), (26) and the changes of coordinates $\Phi^{-1}$ and $U$ with the following parameters value:

$$
k_{d}=1, k_{i}=10 \text { and } K_{\lambda}=0.1,
$$

which comply with statements of Theorem 2 .

\section{A. Experiment 1: Decomposition Highlighting}

1) Cost function: For this experiment, the cost function is purely academic and defined as

$$
J(\varphi)=\frac{1}{2}\left(\varphi_{1}-\varphi_{2}-\mathcal{C}^{\star}\right)^{2},
$$

where $\mathcal{C}^{\star} \in \mathbb{R}$ is a parameter. $J$ has been chosen for its straightforward expression in the $z$-coordinates since

$$
J_{z}(\mathcal{C})=\frac{1}{2}\left(\mathcal{C}-\mathcal{C}^{\star}\right)^{2} .
$$

Therefore, asymptotic value of $\mathcal{C}$ is $\mathcal{C}^{\star}$ for which minimum of $J_{z}$ is reached. Note that $J_{z}$ trivially satisfied Assumption 1.

2) Experiment environment: The experiment is divided into three phases:

- Phase (1): $t \in[0 ; 1[\mathrm{~s}$. Initially at $0 \mathrm{C}$, at $t=0 \mathrm{~s}$ the charge reference $Q_{r}$ is set to $264 \mathrm{mC}$ for a load value of $R=20 \Omega$. Parameter $\mathcal{C}^{\star}$ is set at $0 \mathrm{~Wb}$.

- Phase (2): $t \in[1 ; 2[\mathrm{~s}$. At $t=1 \mathrm{~s}$, load magnitude switches to $R=5 \Omega$ and $\mathcal{C}^{\star}$ is still at $0 \mathrm{~Wb}$.

- Phase (3): $t \in[2 ; 3]$ s. At $t=2 \mathrm{~s}, \mathcal{C}^{\star}$ is set at $5 \times 10^{-3} \mathrm{~Wb}$.

Because of decoupling of $\Sigma$ in $\Sigma_{Q}$ and $\Sigma_{\mathcal{C}}$, value of $\mathcal{C}$ is expected to remain unchanged when Phase (2) occurs whereas value of $Q$ and $\varphi_{T}$ are expected to remain unchanged when Phase (3) occurs.

3) Results: Results of Experiment 1 are given by Fig. 8. Subplot 1 depicts charge $Q$ with the reference $Q_{r}$, Subplot 2 depicts fluxes through $L_{1}$ and $L_{2}$ while Subplot 3 displays $\mathcal{C}$ with the reference $\mathcal{C}^{\star}$.

We see on Fig. 8 that when the load changes value $(t=1 \mathrm{~s})$, the flow distribution $\mathcal{C}$ is almost not impacted. In the same way, when the flow distribution is varying $(t=2 \mathrm{~s})$, the load charge $Q$ is almost not impacted. An extremely small overshoot can be
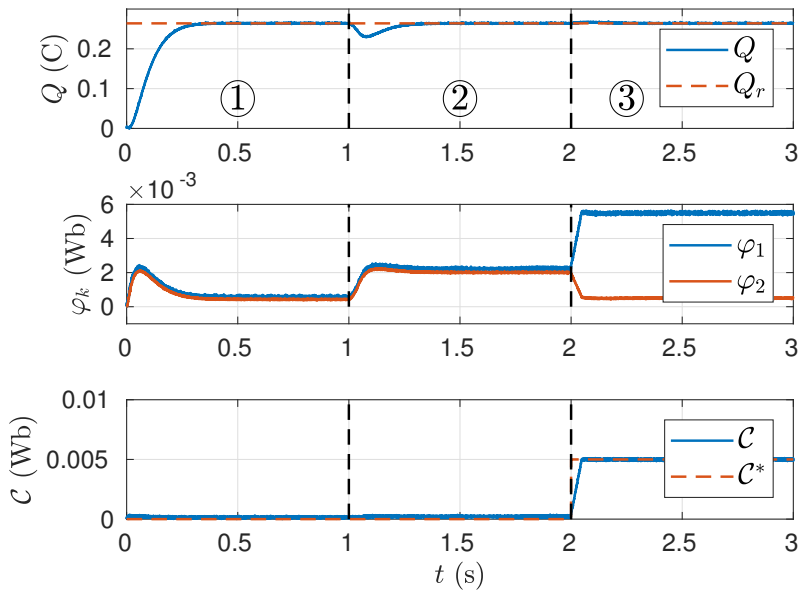

Fig. 8: Time results for Exp. 1.

observed on $Q$. This might come from uncertainties on $L$ and ESR of inductors. Magnitude of this overshoot proves that $\Sigma_{Q}$ and $\Sigma_{\mathcal{C}}$ are almost fully (robustly) disconnected as shown by Fig. 3. This validates robustness of the approach.

\section{B. Experiment 2: minimization of power losses}

Experiment 2 aims providing a meaningful practical application of paper result. Minimization of power losses is considered for defining the cost function and an unknown load variation is taken into account.

1) Experiment environment: The experiment is divided into two phases:

- Phase (1): $t \in[0 ; 1[\mathrm{~s}$. Initially at $0 \mathrm{C}$, at $t=0 \mathrm{~s}$ the charge reference $Q_{r}$ is set to $264 \mathrm{mC}$. During this phase, the load value is $R=20 \Omega$.

- Phase (2): $t \in[1 ; 2] \mathrm{s}$. At $t=1 \mathrm{~s}$, magnitude of the load is instantly changed to $R=5 \Omega$.

2) Cost function: In [19], it is stated that power losses in $k$-th converter can be expressed as the following quadratic function in terms of converter current $i_{k}$ :

$$
p_{k}\left(i_{k}\right)=r_{1, k} i_{k}^{2}+r_{2, k} i_{k},
$$

where $r_{1, k}$ and $r_{2, k}$ are constants depending on electrical components and come from a finer model than (1) (see [19] for numerical values). We consider minimization of overall losses, i.e. the sum of $p_{k}$, as a secondary objective, so that the cost function reads

$$
J(\varphi)=\sum_{k=1}^{m} p_{k}\left(\frac{\varphi_{k}}{L_{k}}\right)=\varphi^{\top} \operatorname{diag}\left\{k_{1}\right\} \varphi+k_{2}^{\top} \varphi,
$$

where

$$
\operatorname{diag}\left\{k_{1}\right\}=\operatorname{diag}\{L\}^{-1} \operatorname{diag}\left\{r_{1}\right\} \operatorname{diag}\{L\}^{-1},
$$




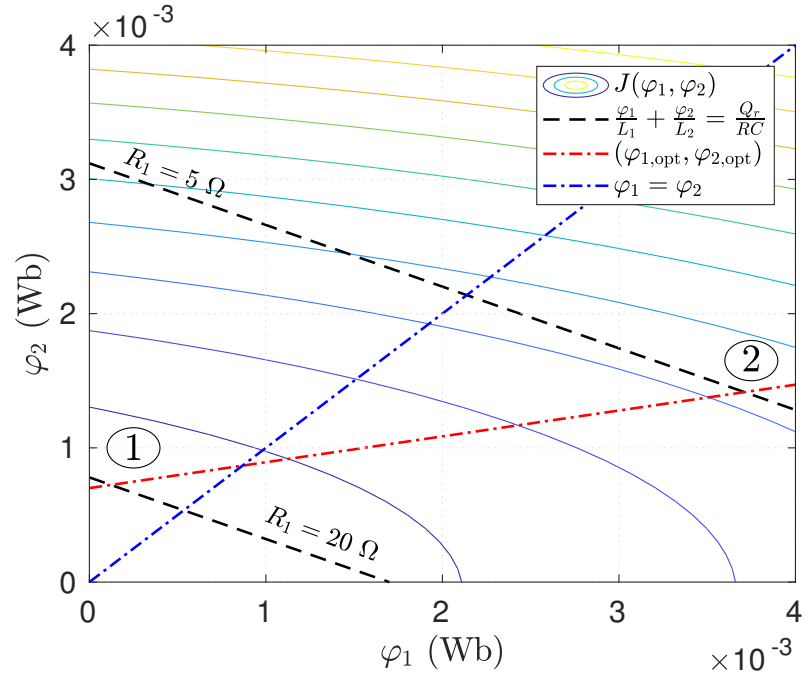

Fig. 9: Cost function levels and optimal repartition

and $k_{2}^{\top}=r_{2}^{\top} \operatorname{diag}\{L\}^{-1}$.

Controller gains are selected as follows:

$$
k_{1}=\left[\begin{array}{l}
0.1623 \\
1.8343
\end{array}\right] \times 10^{5} \text { and } k_{2}=\left[\begin{array}{c}
130.7 \\
27.7
\end{array}\right] .
$$

Fig. 9 depicts cost function $J$ levels (elliptical sections) as well as admissible equilibriums when $Q=Q_{r}$ for $R=20 \Omega$ and $R=5 \Omega$ (black dashed lines). The blue dashed line $\varphi_{1}=\varphi_{2}$ is the frontier above which more flux goes through the second coil than through the first one. System trajectory evolves below whenever the opposite relationship holds. The optimal locus as a function of the load are located by the red dashed line. On this line power losses are minimal since flux repartition is optimal. Intersection between red dashed line and the black one give the equilibrium point that the closed-loop is expected to reach. Indeed, at the intersection, we ensure that $Q=Q_{r}$ and $J$ is minimal.

3) Experimental results: Fig. 10 depicts results of Experiment 2. Subplot 1 depicts charge $Q$ with the reference $Q_{r}$, Subplot 2 depicts the flux through both inductors while Subplot 3 displays duty cycle of each converter.

Benefit of this last control law is that when the load magnitude changes, the closed-loop converge to the equilibrium point satisfying $Q=Q_{r}$ and minimizing the cost function $J$. Indeed we can see that for the first phase $(R=20 \Omega)$, there is more flux passing through the second coil than through the the first one: it corresponds to the (1) of Fig. 9. Yet, for phase $2(R=5 \Omega)$, minimization of power losses gives the priority to the first coil: point (2) of Fig. 9 is therefore recovered.
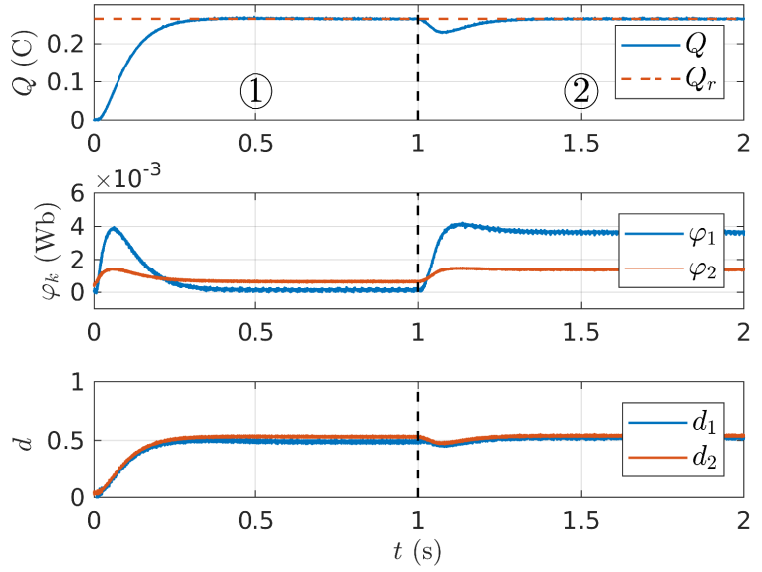

Fig. 10: Time results for Exp. 2: energy variables.

\section{CONCLUSION}

In this paper, charge dynamics has been separated from flux distribution in the Hamiltonian framework. This separation is related to Casimir functions and translates control objectives. The foremost objective of charge regulation is disconnected from the corresponding secondary objective, corresponding to current repartition and related to Casimir function. Once the separation is done by a change of coordinates, control design can be decomposed in two parts. On the one hand, for the charge regulation, we designed a load independent controller performing an integral action on a non passive output. On the other hand, the control of Casimir functions is designed to minimize the cost function by considering it as the virtual energy of the closed-loop. Experiments have been done in order to highlight the relevance of the proposed control scheme for solving meaningful practical problem of minimization overall losses in the electrical circuit.

Further research will mainly focus an three points. Firstly, we want to integrate other converters (for instance boost converters) in parallel interconnection of converters. This induces non-linearities in the model. Secondly, we want to consider input constraints as duty-cycles are constrained to live in compact set $[0,1]^{m}$. How to preserve the decoupling between dynamics of flux repartition and output voltage is an open question. Thirdly, robustness with respect to large serial resistance could be interesting. An adaptive way to recover the value of those resistances could be relevant. Finally, dealing with non constant load impedance, like Constant Power Load (CPL), is an practically relevant direction to extend this work. 


\section{REFERENCES}

[1] V. Joseph Thottuvelil and George C. Verghese. Analysis and control design of paralleled dc/dc converters with current sharing. In Proceedings of APEC 97 - Applied Power Electronics Conference, volume 2, pages 638-646 vol.2, Feb 1997.

[2] Juanjuan Sun, Yang Qiu, Bing Lu, Ming Xu, Fred C. Lee, and W. C. Tipton. Dynamic performance analysis of outer-loop current sharing control for paralleled dc-dc converters. In Twentieth Annual IEEE Applied Power Electronics Conference and Exposition, 2005. APEC 2005., volume 2, pages 1346-1352 Vol. 2, March 2005.

[3] Yuehui Huang and Chi K. Tse. Circuit theoretic classification of parallel connected dc /dc converters. IEEE Transactions on Circuits and Systems I: Regular Papers, 54(5):1099-1108, May 2007.

[4] Jean-François Trégouët and Romain Delpoux. Parallel interconnection of buck converters revisited. IFAC-PapersOnLine, 50(1):15792 - 15797，2017. 20th IFAC World Congress.

[5] Arjan van der Schaft and Dimitri Jeltsema. Port-Hamiltonian Systems Theory: An Introductory Overview, volume 1. now Publishers Inc., 2014.

[6] Hebertt Sira-Ramirez, Rafael A. Perez-Moreno, Romeo Ortega, and Mauricio Garcia-Esteban. Passivity-based controllers for the stabilization of dc-to-dc power converters. Automatica, 33(4):499 - 513, 1997.

[7] Gerardo Escobar, Arjan J. van der Schaft, and Romeo Ortega. A hamiltonian viewpoint in the modeling of switching power converters. Automatica, 35(3):445 - 452, 1999.

[8] Romeo Ortega, Arjan van der Schaft, Bernhard Maschke, and Gerardo Escobar. Interconnection and damping assignment passivity-based control of port-controlled hamiltonian systems. Automatica, 38(4):585 - 596, 2002.

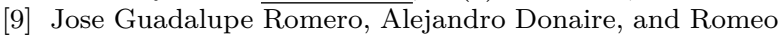
Ortega. Robust energy shaping control of mechanical systems. Systems \& Control Letters, 62(9):770 - 780, 2013.

[10] Romeo Ortega and Jose Guadalupe Romero. Robust integral control of port-hamiltonian systems: The case of nonpassive outputs with unmatched disturbances. Systems \& Control Letters, 61(1):11 - 17, 2012.

[11] Alejandro Donaire and Sergio Junco. On the addition of integral action to port controlled hamiltonian systems. Automatica, 45(8):1910 - 1916, 2009.

[12] Arjan van der Schaft. L2 - Gain and Passivity Techniques in Nonlinear Control. Springer-Verlag London, 2000.

[13] Bernhard Maschke and Arjan van der Schaft. Portcontrolled hamiltonian systems: Modelling origins and systemtheoretic properties. IFAC Proceedings Volumes, 25(13):359 - 365, 1992. 2nd IFAC Symposium on Nonlinear Control Systems Design 1992, Bordeaux, France, 24-26 June.

[14] Shiguo Luo, Zhihong Ye, Ray-Lee Lin, and Fred C Lee. A classification and evaluation of paralleling methods for power supply modules. In 30th Annual IEEE Power Electronics Specialists Conference. Record.(Cat. No. 99CH36321), volume 2, pages 901-908. IEEE, 1999.

[15] Eduardo D. Sontag. Remarks on stabilization and inputto-state stability. In Proceedings of the 28th IEEE Conference on Decision and Control, pages 1376-1378 vol.2, Dec 1989.

[16] Hassan K. Khalil. Nonlinear Systems. Pearson Education. Prentice Hall, third edition edition, 2002.

[17] Mrdjan Jankovic, Rodolphe Sepulchre, and Petar V Kokotovic. Constructive lyapunov stabilization of nonlinear cascade systems. IEEE transactions on automatic control, 41(12):1723-1735, 1996

[18] Romain Delpoux, Jean-Fraçois Trégouët, Jean-Yves Gauthier, and Cyril Lacombe. New framework for optimal current sharing of nonidentical parallel buck converters. IEEE Transactions on Control Systems Technology, 27(3):1237-1243, 2018.

[19] Jean-François Trégouët, Romain Delpoux, and Jean-Yves Gaulthier. Optimal secondary control for dc microgrids. In 2016 IEEE 25th International Symposium on Industrial Electronics (ISIE), pages 510-515, Santa Clara, CA, June 2016.

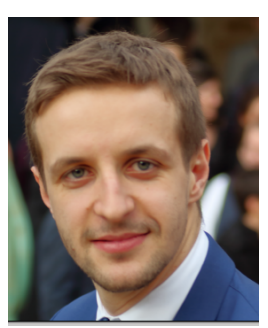

Jérémie Kreiss obtained his Ph.D in Control Systems in 2019 from the University of Lyon, France, working on control allocation for electrical drives and power electronics systems. $\mathrm{He}$ is currently a research and teaching assistant from Ecole Centrale Lyon (France). His research interests concern control allocation applied to electrical drives and power electronics systems, and constraints management.

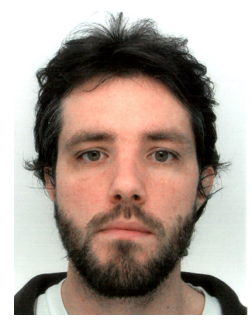

Jean-François Trégouët received his Ph.D. degree from Supaéro (Toulouse, FR) in 2012 after performing his reasearch at LAAS-CNRS (Toulouse, FR). He is now Assistant Professor at INSA-Lyon (FR). He spent several months in various research institutions (Politecnico di Milano (IT), Kyoto University (JP), Curtin University (Perth, $\mathrm{AU})$ ). His main research interests include control design for over-actuated systems, with applications in power electronics.

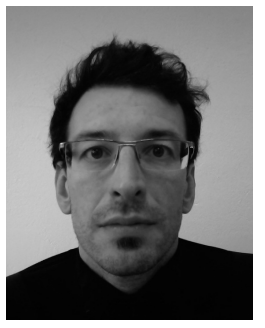

Damien Eberard obtained his Ph.D. in Control Systems from the University of Lyon, and was a Postdoctoral Scholar with the Institute for Mathematics and Computer Science at the University of Groningen, The Netherlands. He is currently Associate Professor at INSA Lyon, Ampère Lab in Lyon. His research interests include control and simulation of networked portHamiltonian systems, and control of linear hyperbolic PDE's. 


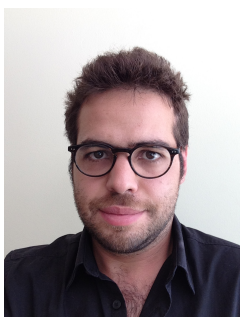

Romain Delpoux received the M.S. degree in Control System and Mechatronics form Chalmers University of Technology, Gothenburg, Sweden in 2009 and the Ph.D degree in control theory, data and signal processing, from Ecole Centrale de Lille, France in 2012. In september 2014, he joined INSA Lyon and the Ampère Lab, France, where he is currently an Associate Professor. His research interrests include control applications for power electronics systems, switching systems and sliding mode control.

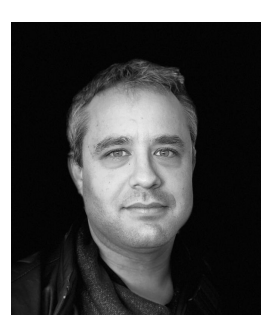

Jean-Yves Gauthier received the Ph.D. degree both from the University of Franche-Comté, France, in 2007. His Ph.D. research fields was about the modeling and the control of magnetic shape memory alloy-based actuators. In 2008, he became an Associate Professor at INSA Lyon, France, working on semiactive vibration control using piezoelectric devices. Since 2012, he has been working at Ampère Lab, France. His current research interests include the control of power electronic devices and electrical motor drives.

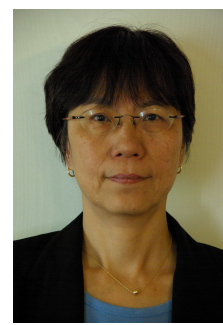

systems.
Xuefang Lin-Shi received the Ph.D degree in applied computer science and automatic in 1992 from INSA Lyon (Institut National des Sciences Appliquées de Lyon), France. Since 1993, she has been with the Electrical Engineering department of INSA Lyon, where she is currently a professor. She is now with Ampère Laboratory in Lyon. Her research interests concern control applied to electrical drives and power electronics 